\title{
Theory of the Navier-Stokes Equations
}




\section{This page is intentionally left blank}




\section{Theoril of the \\ Navier-Stokes Equations}

Editors

J. G. Heywood

University of British Columbia, Canada

K. Masuda

Tôhoku University, Japan

R. Rautmann

Universität-GH Paderborn, Germany

V. A. Solonnikov

Steklov Mathematical Institute, St. Petersburg, Russia 
Published by

World Scientific Publishing Co. Pte. Ltd.

P O Box 128, Farrer Road, Singapore 912805

USA office: Suite 1B, 1060 Main Street, River Edge, NJ 07661

UK office: 57 Shelton Street, Covent Garden, London WC2H 9HE

\section{Library of Congress Cataloging-in-Publication Data}

Theory of the Navier-Stokes equations / editors, J.G. Heywood ... [et al.].

p. cm. -- (Series on advances in mathematics for applied sciences ; vol. 47)

Proceedings of the third international conference held at Oberwolfach, Germany. Includes bibliographical references.

ISBN 9810233000 (alk. paper)

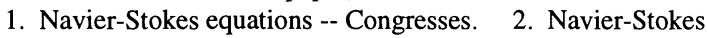

equations -- Numerical solutions -- Congresses. I. Heywood, J. G.

(John Groves), 1940- II. Series.

QA929.T48 1997

532'.0533'01515353 -- dc21

\section{British Library Cataloguing-in-Publication Data}

A catalogue record for this book is available from the British Library.

Copyright () 1998 by World Scientific Publishing Co. Pte. Ltd.

All rights reserved. This book, or parts thereof, may not be reproduced in any form or by any means, electronic or mechanical, including photocopying, recording or any information storage and retrieval system now known or to be invented, without written permission from the Publisher.

For photocopying of material in this volume, please pay a copying fee through the Copyright Clearance Center, Inc., 222 Rosewood Drive, Danvers, MA 01923, USA. In this case permission to photocopy is not required from the publisher.

This book is printed on acid-free paper. 
This Volume is dedicated to the memory of

Professor Anatoli P. Oskolkov

$(† 1995)$

and

Professor Valeriy Y. Rivkind

$(\dagger 1996)$ 


\section{This page is intentionally left blank}




\section{Introduction}

The articles in this volume constitute the proceedings of the third international conference held at Oberwolfach under the title "The Navier-Stokes Equations: Theory and Numerical Methods". The organizers were John Heywood (Vancouver), Kyuya Masuda (Tokyo), Reimund Rautmann (Paderborn), and Vsevolod A. Solonnikov (St. Petersburg).

These articles make important contributions to a wide variety of topics in the Navier-Stokes theory: general boundary conditions, flow exterior to an obstacle, conical boundary points, the controllability of solutions, compressible flow, non-Newtonian flow, magneto-hydrodynamics, thermal convection, the interaction of fluids with elastic solids, the regularity of solutions, and Rothe's method of approximation.

The article by Gerd Grubb (Copenhagen) offers a general classification of boundary conditions for the Navier-Stokes equations, and a unified treatment of the corresponding initial boundary value problems by the reduction of degenerate parabolic problems to pseudodifferential problems, and the subsequent application of a general theory for these pseudodifferential problems.

The article by Reinhard Farwig (Darmstadt) and Hermann Sohr (Paderborn) gives a new treatment of the problem of steady flow past an obstacle, by a method of weighted $L_{p}$-estimates that avoids explicit integral representations. In the three-dimensional case, they provide new proofs and extensions of the famous theory of "physically reasonably solutions" of Finn, Babenko, and Galdi. The article by Hans-Christoph Grunau (Bayreuth) begins the development of an $L_{p}$-approach to the stability of such physically reasonable solutions. The article by Hideo Kozono (Nagoya) and Masao Yamazaki (Tokyo) addresses the particular difficulties that are encountered in treating flow in exterior domains when the prescribed limit at infinity is zero.

Control problems are pioneered in an article by Andrei V. Fursikov (Moscow), where the controllability of three-dimensional flow by Dirichlet boundary conditions is investigated. For arbitrarily given initial values, it is proven that there exist boundary values which will bring the solution to rest in finite time. In another unusual article, Michael Ruzicka (Bonn) and Jens Frehse (Bonn) investigate the regularity of steady solutions in dimensions 5 and greater, motivated by a number of surprising analogies with the famous global existence problem for three-dimensional nonstationary flow.

Two of the articles presented here concern the extension of classical NavierStokes theory to fluids with more general constitutive laws. Antonin Novotny (Toulon) contributes to the global existence theory for steady solutions of the compressible isentropic Navier-Stokes equations, using a method of supersonic decomposition. Mariarosaria Padula (Ferrara) and Adelia Sequeira (Lisbon) 
prove the well-posedness of the boundary value problem of a vector transport equation that arises in the study of non-Newtonian fluids.

Three of the articles concern the coupling of the Navier-Stokes equations with other physical effects. Martin Rumpf (Freiburg) has analyzed the interaction between a thick elastic obstacle and a viscous fluid. Yoshiyuki Kagei (Fukuoka) has investigated the global attractor for two-dimensional Bénard convection, using governing equations that include the heating effect of viscous dissipation. Maria Schonbek (Santa Cruz) has investigated the decay properties of magneto-hydrodynamic flow, showing that if the energy of the magnetic field is non-oscillating, then the energy of the velocity field decays to zero.

Three of the articles presented here take up fundamental issues concerning the linear Stokes equations. Hermann Sohr (Paderborn) and Maria SpecoviusNeugebauer (Paderborn) give a new theory of the exterior Stokes problem using homogeneous Sobolev spaces. This is based in part on a new decomposition theorem for these spaces. Paul Deuring (Magdeburg) gives a new approach to the regularity of solutions of the Stokes equations in the neighborhood of conical boundary points. In particular, he supplies $L_{p}$-estimates. Werner Varnhorn (Dresden) constructs a solution of the Stokes resolvent equations by methods of hydrodynamic potential theory. This is based on a new explicit representation of the fundamental tensor.

Finally, in the direction of numerical applications, Reimund Rautmann (Paderborn) presents an article on a new convergence result for Rothe's method of approximating the Navier-Stokes equations. - In this place we have to thank Kerstin Wielage for fitting together all the contributions written in heterogeneous codes.

John Heywood, Kyuya Masuda, Reimund Rautmann, Vsevolod A. Solonnikov 


\section{CONTENTS}

Introduction vii

The 3D Stokes Systems in Domains with Conical Boundary Points . . . 1 P. Deuring

Weighted Estimates for the Oseen Equations and the Navier-Stokes

Equations in Exterior Domains .................... 11

R. Farwig and H. Sohr

On Boundary Zero Controllability of the Three-Dimensional

Navier-Stokes Equations . . . . . . . . . . . . . . . .

A.V. Fursikov

Nonhomogeneous Navier-Stokes Problems in $L_{p}$ Sobolev Spaces

over Exterior and Interior Domains ...................

G. Grubb

$L^{p}$-Decay Rates for Strong Solutions of a Perturbed

Navier-Stokes System in $\mathbb{R}^{3} \ldots \ldots \ldots \ldots \ldots \ldots \ldots \ldots$ H.Ch. Grunau

On Two-Dimensional Equations of Thermal Convection in the

Presence of the Dissipation Function $\ldots \ldots \ldots \ldots \ldots \ldots \ldots \ldots$

Y. Kagei

Exterior Problem for the Navier-Stokes Equations, Existence,

Uniqueness and Stability of Stationary Solutions ............

H. Kozono and M. Yamazaki

On Decay Properties of Solutions to Stokes System in

Exterior Domains . . . . . . . . . . . . . . . . . . . . . . . . . . . . . 99

P. Maremonti and V.A. Solonnikov

Compactness of Steady Compressible Isentropic Navier-Stokes

Equations via the Decomposition Method (the Whole 3-D Space) ...

A. Novotny

A Note on a Vector Transport Equation with Applications to

Non-Newtonian Fluids.

M. Padula and A. Sequeira 
Convergence Rates in $H^{2, r}$ of Rothe's Method to the

Navier-Stokes Equations . . . . . . . . . . . . . . . . . .

R. Rautmann

On Equilibria in the Interaction of Fluids and Elastic Solids $\ldots \ldots \ldots 136$

M. Rumpf

Regularity for Steady Solutions of the Navier-Stokes Equations . . . 159

M. Rüžčka and J. Frehse

Decay of Non-Oscillating Solutions to the Magneto-Hydrodynamic

Equations . . . . . . . . . . . . . . . . . . . . . . . . . . . . . . 179

M. E. Schonbek

The Stokes Problem for Exterior Domains in Homogeneous

Sobolev Spaces .................................... 185

H. Sohr and M. Specovius-Neugebauer

Boundary Value Problems and Integral Equations for the

Stokes Resolvent in Bounded and Exterior Domains of $\mathbb{R}^{n} \ldots \ldots \ldots 206$

W. Varnhorn

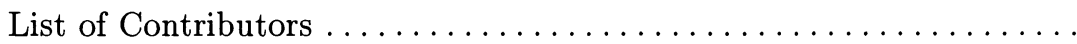

